\title{
Analytical modelling solution of producer mobility support scheme for named data networking
}

\author{
Muktar Hussaini, Shahrudin Awang Nor, Amran Ahmad \\ InterNetWorks Research Laboratory, School of Computing, Universiti Utara Malaysia, Malaysia
}

\begin{tabular}{l} 
Article Info \\
\hline Article history: \\
Received Oct 3, 2018 \\
Revised Apr 2, 2019 \\
Accepted Apr 14, 2019 \\
\hline
\end{tabular}

\section{Keywords:}

Information-centric networking

Mobility approach

Mobility support scheme

Named data networking

Producer mobility

\begin{abstract}
Named Data Networking (NDN) is a clean-slate future Internet architecture proposed to support content mobility. However, content producer mobility is not supported fundamentally and faces many challenges such as high handoff latency, signaling overhead cost and unnecessary Interest packet losses. Hence, many approaches indirection-based approach, mapping-based approach, locator-based approach and control/data plane-based approach were proposed to address these problems. Mapping-based and control/data plane-based approach deployed servers for name resolution services to provide optimal data path after the handoff, but introduces high handoff latency and signaling overhead cost. Indirection-based and locator-based approach schemes provide normal handoff delay but introduce sub-optimal or triangular routing path. Therefore, there is needs to provide substantial producer mobility support that minimizes the handoff latency, signaling cost and improve data packets delivery via optimal path once a content producer relocates to a new location. This paper proposed a scheme that provides optimal data path using mobility Interest packets and broadcasting strategy. Analytical investigation result shows that our proposed scheme outperforms existing approaches in terms of handoff latency, signaling cost and path optimization.
\end{abstract}

Copyright $(0) 2019$ Institute of Advanced Engineering and Science. All rights reserved.

\section{Corresponding Author:}

Muktar Hussaini,

InterNetWorks Research Laboratory

School of Computing,

Universiti Utara Malaysia,

06010 UUM Sintok Kedah Darul Aman, Malaysia.

Email: elhussenkaz@yahoo.com

\section{INTRODUCTION}

Named Data Networking (NDN) is an architecture completely re-design for the proposed future Internet that evolved principally from the successes of the current Internet that are IP architecture [1, 2]. The architecture was proposed to supplement and replace IP architecture due to the high demand of network content such as mobile data [3], high bandwidth and mobility [4] latency in IoT network [5], mobility management and vehicular networks VANET [6]. To achieve routing and forwarding of packets, NDN nodes uses named-base routing and possesses two different types of packets, namely Interest and Data packets. The node can be represented as client consumer, producer or a router, that maintains three data structure Pending Interest Table (PIT), Forwarding Information Base (FIB) and Content Store (CS), determines when and where to forward Data and Interest packets [7]. PIT records and store any incoming Interest information, FIB maintained the Forwarding Strategy and decide when and where to forward Interest and CS is a temporary cache of data stored based on the NDN caching policy.

Fundamentally, NDN was proposed to support content mobility, thus proposed as a consumerdriven network. When content consumer relocates to a new domain or access point, it has the ability to send pending Interest packets that are not satisfied with relevant data. The caching capability of NDN suppressed 
the problem of consumer mobility by supplementing of cached data to support node mobility [8, 9]. However, many challenges aroused especially for the support of content producer [10, 11], due to the consumer-driven nature of NDN architecture. Therefore, the in-depth review on mobility support concept and approaches was discovered that some researchers classified existing producer mobility support concepts, schemes, and approaches into different classes based on their fundamental design, for easy identification and analysis of their strengths and weaknesses. Hermans, Ngai, and Gunningberg [12] proposed a location-based concept and ascertain that to provide a concrete solution for producer mobility, a content identifier, and content locator decoupling is necessary. Added that, many challenges were raised to solve the mobility support problems such as preferable namespace to be used for the content identifier and content locator, provision of session continuity, how to resolve identifiers and locators using mobility anchor or explicit identifier. In addition, Zhu, Afanasyev, and Zhang (2013) presented three different conceptual schemes for a new perspective to support mobility in NDN, that is, using Mobile Producer (MP) storage to fetch data in the absence of producer, using intermediate nodes such as DNS servers, anchor, rendezvous and Interest broadcasting for mapping between content identifier and location [13, 14]. Saxena et al. [11] further classified producer mobility support approach as mapping-based approach and locator-free approach. Producer mobility support approaches were grouped into Rendezvous-point Based Mobility Approach (RBMA) or home repository and Indirection-point Based Mobility Approach (IBMA) and Location-Based Mobility Approach [15, 16].

The problem that affects producer mobility in NDN is as a result of using named based routing (NBR) and hierarchical naming structure to aggregate route announcements. For a mobile content producer, a new route needs to be announced and propagated to replace old routing information in the FIB, causing a similar problem as in mobile IP [1]. Besides, producer mobility in NDN caused a serious problem that offers long handoff latency and unnecessary Interest packet losses on transmission towards the old location of the producer. Moreover, the content producer mobility faces many problems similar to mobility in IP architecture, such as routing table size scaling problem [13], introduces significant scalability challenge [17, 18], offers long handoff delay and unnecessary Interest packet losses on transmission towards the old location of the content Producer $[15,16]$, for a large network domain the frequent routing update cost high bandwidth utilization $[17,19]$ and the problems of tunneling and triangular routing. The same problems arise in the case of CCN as NDN rooted in it $[20,21]$. Therefore, this paper proposed a substantial analytical solution of producer mobility support that minimizes the handoff latency, signaling cost and improved data packets delivery via optimal path once a content producer relocates to a new location.

The paper is organized into seven sections, apart from introduction and statement of the problem, section two discussed the related works done to improved producer mobility for NDN. Section three is the proposed scheme that used mobility Interest packets and broadcasting strategy, then formulation of the analytical model. Section five discussed the design and implementation of the analytical model. Section six is the result analysis of the proposed scheme for the performance evaluation of handoff latency, signaling cost and path optimization, lastly, section seven is the conclusion of this paper.

\section{RELATED WORK}

The mobility support approach in NDN for both consumer and producer were classified as partial separation (PS) and temporary separation (TS) [10], Location Resolution Approach (LRA) [22] IndirectionBased Mobility Approach (IBMA), Mapping-Based Mobility Approach (MBMA), Locator-Based Mobility Approach (LBMA) and Control/Data Plane-Based Approach (CDPBMA)

The Mapping-Based Mobility Approach (MBMA) is a technique also called DNS-like, rendezvous or Indirection server approach, adopted by some researchers [23]-[28] to support producer mobility in NDN. The schemes proposed was depended on a DNS server, rendezvous point, and mapping server to keep and processes mapping track of content identifier and the current location of the mobile producer. Jiang et al., provide a solution to the content producer mobility in NDN using DNS-like mapping approach and adopt forwarding hint to explore the benefit of NDN in supporting mobility [25]. Another mapping-based approach mobility support scheme was proposed by Gao and Zhang [23] to solve the producer mobility problem in NDN without tampering the original NDN architecture [23]. The scalable mobility management (SMM) scheme adopts three different separation mechanisms, access/core separation, locator/ID separation, and management/routing separation. In general, the mapping-based approach provides an optimal routing path for data packets delivery after the handoff. However, the approach has high signaling cost and high handoff latency, due to signaling exchange for location update between the producer, the consumer and mapping server. Moreover, partial modification of NDN architecture that may requires additional infrastructure such as mapping server and distributed servers for a large network. 
The Indirection-based Mobility Approach (IBMA) is a technique derived from the MIPv4 and MIPv6 mobility solution concept to support the mobile producer in NDN architecture [11]. A home agent or home router was provided to maintain the binding information between content prefix content location and redirect the Interest packets using the tunnel to the new location or PoA of the mobile producer [29, 30]. Lee et al. also proposed an indirection approach similar to [30] that uses the home domain Content Router $\left(\mathrm{CR}_{\mathrm{h}}\right)$ and intermediate Content Routers $(\mathrm{CR})$ to handle Producer mobility. The mobile producer always sends a prefix update to $\mathrm{CR}_{\mathrm{h}}$ about its movement to a new location; also announce the prefix to the new CR [29]. A proposed protocol was design, evaluated and benchmark against purely indirection approach and traditional CCN architecture. The names were used to update the home router and old PoA about the mobility event of content producer [31, 32]. For the normal process when the producer does not move, all interest packets were forwarded through the home router using static FIB. Yan et al. proposed a distributed mobility management scheme as a solution for both consumer and producer mobility in NDN architecture [33], and all mobile IP architecture supported by NDN overlay [28]. The distributed mobility management handover scheme supports both consumer and producer mobility which minimized handoff latency and routing update cost or signaling overhead cost. In general, the indirection-based approach provides normal handoff latency and signaling cost. However, the approach has a triangular routing problem.

Rao, Luo et al. [16] and Rao Gao et al. [15] proposed Locator-Based producer mobility scheme by adding a locator to each AR (Access Router) in NDN network as unique topological layer, also extend the functionality of AR with capability of catching, forwarding Interest packets on behalf of the source and added a field in the original NDN packet. FIB entries that contain the only list of interfaces is modified to include a list of mobility status and locator. The scheme was called locator/identifier separation-based approach [19] where the mapping of producer's content prefix that serves as an identifier and its current location take place by home router. Location-aware on-demand multipath protocol based on NDN MANETs was proposed by Asif and Kim [34] to solve the intermittent connectivity, low battery power, data redundancy, packet flooding that affect network performance due to the nature of broadcast nature of wireless mobile ad-hoc network in NDN. In the study of Azgin, Ravindran and Wang an anchor chain on-demand mobility support solution for ICN was proposed to manage producer mobility and handoff processes to avoid data loss [35]. The architecture used decentralized micro-level resolution system and chained distributed anchors that provide forwarding functionalities to help for the efficient packets forwarding to the mobile producer after handoff [35]. In general, LBMA provides normal handoff latency, signaling cost and partial modification of NDN architecture. However, the approach did not consider path optimization after the handoff and the central node (Home repository, Anchor, Access router) utilization can be resulted in path stretching and serve as a single point of failure.

The Control/Data Plane-based Mobility Approach (CDBMA) is a technique based on the control plane and data plane separation to support mobile producer. The control plane used a server that serves as controller [36] or resource handler [37] to control the process of mobility signaling during the handoff. To reduce the handoff latency and minimized the signaling overhead Ren et al. proposed a producer mobility management scheme using Software Define Controller (SDC) to improve the performance of [37] scheme. The basic idea of the proposed scheme is to separate control from data plane as in [36, 37], updating routing tables entries and configures the relevant content routers by the SDC [38]. Joao et al. proposed Controllerbased Routing Scheme for NDN to solve the scalability problems caused by content mobility that intensifies a high number of contents in different locations. The scheme splits content identity and content location from name prefix to facilitate content mobility [39]. However, the scheme does not ensure path optimization after the handoff and when producer mobility rate increases; the overhead will increase and degrade the efficiency. In general, CDBMA provides low packets delivery cost, However, the approach is more of software-defined networking architecture, which is not suitable with NDN architecture and the handoff latency and signaling cost was very high due to the consumer, producer querying processes to the resolution handler server and entire FIBs update.

\section{PROPOSED PRODUCER MOBILITY SUPPORT SCHEME}

Content producer movement in NDN causes some problems that need to be addressed, such problems are high handoff latency or delay, high signaling cost, routing path sub-optimization and unnecessary Interest packet lost. When producer moved to new PoA, the consumer, on the other hand, will continue sending Interest packets via the old path, towards the earlier location of the producer as normal transmission, without knowledge of the producer movement. The PoA that previously attached to the producer will continuously drop the packets. Even though caching plays an important role in the NDN network, but there are certain consumer's requests that are having many numbers of data chunks. Real-time communication that has less priority in caching, or the unsatisfied consumer request, that will make the 
content consumer keep on sending Interest for the unsatisfied request. The communication is interrupted due to the movement of the producer and the NDN network lack knowledge of producer mobility. However, the transmission can only be continued after the accomplishment of FIB entries update for the intermediate nodes involved, that is when the long handoff latency happened.

\subsection{Mobility interest package}

In addition, a new packet Mobility Interest (MI) will be introduced to carry binding information for the content identifier, old and new location of mobile producer, to update the immobile anchor router. The MI packet is similar in general purpose with different functions and format to the forwarding hint $[12,16]$, binding update $[12,29]$, mobility management packet $[19,26,31]$, traced and tracing Interest [40] etc. However, the packets are differed based on the different schemes and techniques.

\subsection{Broadcast strategy}

When mobile producer relocates to new PoA there is need to update the network about the new name prefix of producer's new location. Zhu et al. [13] in NDN technical report that highlighted the new perspective on mobility support in NDN suggested that the mapping between content identifier and content locator can be provided by the broadcasting method of using intermediate nodes like DNS servers. Added that, Interests are relatively small and NDN is broadcast friendly making it practically possible to broadcast in a restricted domain to track the new location of the producer. For a large network, broadcast overlay spanning can be used [34]. NDN support Interest Broadcasting as the Interest are small in size making it feasible to be broadcasted in the restricted domain to track the location of mobile producer [13]. Figure 1 shows the architecture and mode of operation of the proposed scheme.
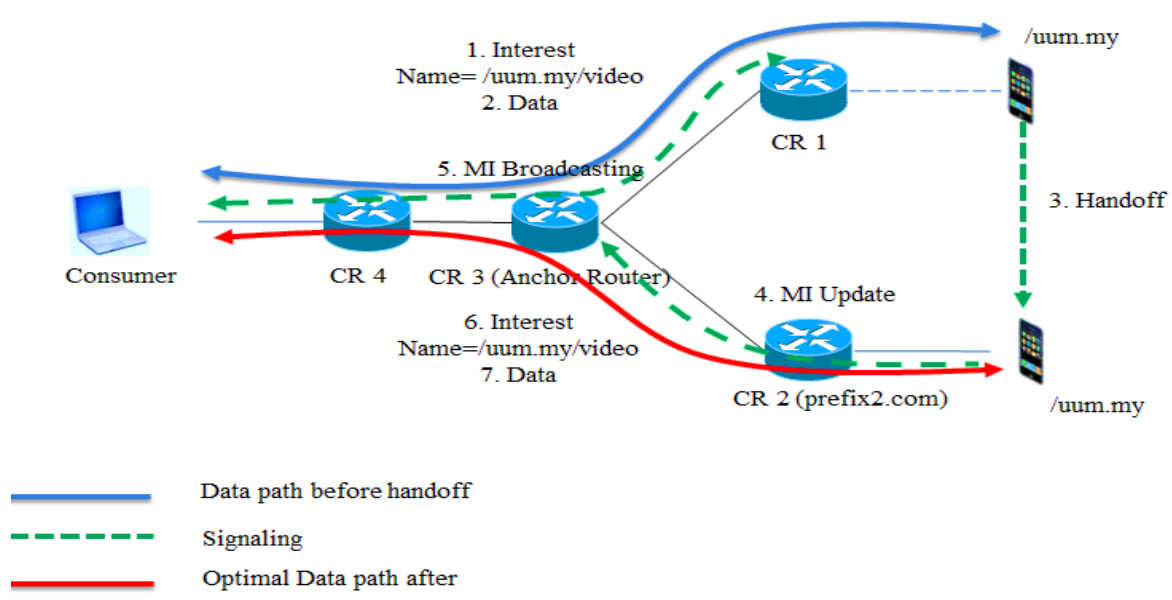

Figure 1. Proposed scheme operational overview

\section{FORMULATION OF ANALYTICAL MODEL}

\subsection{Network analysis model}

To analyze and evaluate the mobility handoff performance of the proposed scheme, we consider a network analysis model as done in $[16,19,23,41]$. The comparison of the proposed scheme and existing were addressed using network analytical model to validate the concept of broadcasting strategy. Handoff latency and cost were been mathematically formulated for MBMA, LBMA, IBMA and CDPBMA to evaluate the concept and address handoff problems. Mathematical models are easier to represents logical behavior between network elements, also covers the bound of research question by showing the borderline of the network behavior or characteristics. The notation, parameters, and values used are presented in Table 1 and the build network analysis model is shown in Figure 2. In the network analysis model presented, the hop count between the content router and consumer or producer, content routers and server represented by $a, b, c$ and $d$ as shown in Table 1. We assumed that the producer has a constant speed. 


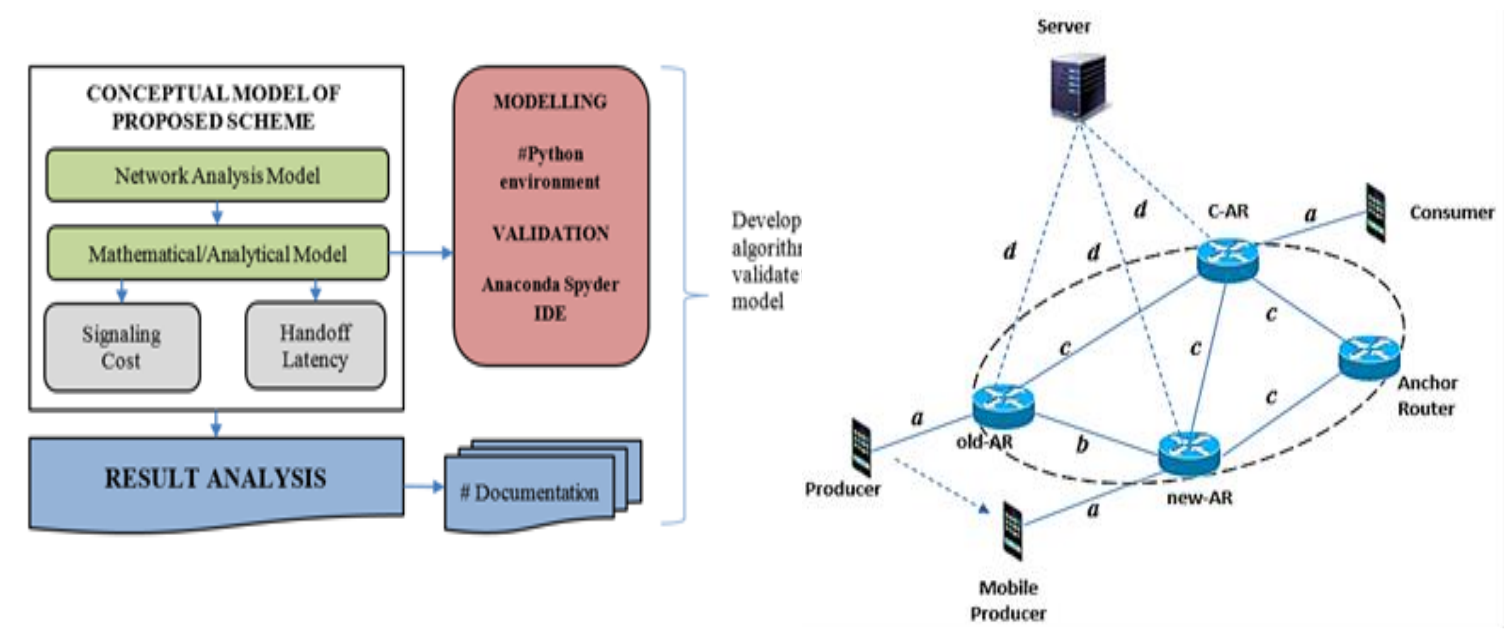

Figure 2. Network analysis model

Table 1. Network analysis model parameters

\begin{tabular}{|c|c|c|c|}
\hline Category & Notation & Parameters & Value \\
\hline \multirow[t]{2}{*}{ Packets } & $\mathrm{S}_{\text {name }}$ & Size of the signaling packet & +16 byte \\
\hline & $\mathrm{S}_{\text {int }}$ & Size of Interest packet & 40 bytes \\
\hline \multirow{3}{*}{ Latency } & $\mathrm{L}_{\mathrm{car}}$ & Transmission latency between consumer to AR & $\mathrm{a}$ \\
\hline & $\mathrm{L}_{\mathrm{sar}}$ & Transmission latency between Server to new AR & $\mathrm{d}$ \\
\hline & $\mathrm{L}_{\mathrm{o}-\mathrm{nar}}$ & Transmission latency between old AR to new AR & $\mathrm{b}$ \\
\hline Signaling & $\mathrm{C}_{\text {par }}$ & Transmission cost hop/packet producer to AR & $\mathrm{a}$ \\
\hline \multirow[t]{4}{*}{ Cost } & $\mathrm{C}_{\mathrm{car}}$ & Transmission cost hop/packet consumer to AR & a \\
\hline & $\mathrm{C}_{\mathrm{o}-\mathrm{nar}}$ & Transmission cost hop/packet old AR to new AR & $\mathrm{b}$ \\
\hline & $\mathrm{C}_{\mathrm{ars}}$ & Transmission cost hop/packet ARs/Anchors & $\mathrm{c}$ \\
\hline & $\mathrm{C}_{\mathrm{s}-\mathrm{nar}}$ & Transmission cost hop/packet new AR to Server & $\mathrm{d}$ \\
\hline
\end{tabular}

\subsection{Handoff latency analysis}

Handoff or handover is a term used interchangeably in mobile networking that refers to the processes where a mobile producer disconnects from current PoA and connects to new neighboring PoA. The period of time that content producer requires from the last Packets (Interest or Data) received via old PoA to the arrival of the first Packets via the new PoA after the handoff is called handoff latency the smaller latency, the better performance of the proposed scheme. (1) and (2) is the wired and wireless link delay between two hops for data, Interest, update or query as used in [19]. Where q is the probability of link failure, $B_{w}$ and $B_{w l}$ are wired and wireless bandwidth, $L_{w}$ and $L_{w l}$ link delay for wired and wireless and $Q_{d}$ is the queuing delay. Their values, $B_{w}=100 \mathrm{Mbps}, B_{w l} 11 \mathrm{Mbps} \mathrm{Ld}_{\mathrm{w}}=2 \mathrm{~ms}$ and $\mathrm{Ld}_{\mathrm{wl}}=10 \mathrm{~ms}$ and $\mathrm{Q}_{\mathrm{d}=5} \mathrm{~ms}$

$$
\begin{aligned}
& L w_{\text {name }}=\left(\frac{S_{\text {name }}}{(B w+L d w+Q d)}\right) \\
& L w l_{\text {name }}=\left(\frac{1+q}{1-q}\right) \times\left(\frac{S_{\text {name }}}{B w l+L d w l}\right)
\end{aligned}
$$

The handoff latency of MBMA can be express as (4). When the content producer moves to new AR, new prefix name should be generated, and the new AR sent an updated prefix to the server. The server performs mapping process to update existing name prefix to generate a forwarding hint or binding update. When round trip time (RTT) elapsed, the content consumer query the server requesting the forwarding hint that will be used to trace the new location of the content producer. The total handoff delay can be generated in (3) together with the two consecutive delays for wired and wires link defined in (1) and (2). 


$$
\begin{aligned}
L_{m b a} & =L_{p n}+L_{p a r}+L_{s a r}+L_{s a r}+L_{c a r}+L_{\text {ars }} \\
& =l_{p n}+a \times L w l_{u p}+2 d \times L w_{u p}+a \times L w l_{\text {int }}+c \times L w_{\text {int }}
\end{aligned}
$$

The handoff latency of LBMA can be express as (4). The handoff process takes place when the producer moves to new AR, after generating new prefix name the new AR sent a location update or binding update to the home router or old AR. When the handoff procedure is over, the content consumer will send the Interest to the old AR that will encapsulate and forward encapsulated Interest to the new AR and the producer. The total handoff delay can be generated as follows:

$$
\begin{aligned}
L_{l b a} & =L_{p n}+L_{\text {par }}+L_{\text {onar }}+L_{\text {car }}+L_{\text {ars }}+L_{\text {onar }} \\
& =l_{p n}+a \times L w l_{u p}+b \times L w_{u p}+a \times L w l_{\text {int }}+c \times L w_{\text {int }}+b \times L w_{\text {int }}
\end{aligned}
$$

The handoff latency of CDBMA is closely related to MBMA and can be express as (5). When the content producer moves to new AR, new prefix name should be generated, and the new AR sent an updated prefix to the control server. The control server performs a mapping process and updates the whole content router (ARs) in the domain. The content consumer can request a control server for the forwarding hint if its AR was not up-to-date. The total handoff latency can be generated as follows:

$$
\begin{aligned}
L_{c d b a} & =L_{p n}+L_{p a r}+3 L_{s a r}+L_{c a r}+L_{a r s} \\
& =l_{p n}+a \times L w l_{u p}+3 d \times L w_{u p}+a \times L w l_{\text {int }}+c \times L w_{\text {int }}
\end{aligned}
$$

The handoff latency of IBMA can be express as (6), is also like that of LBMA, but the binding update is used to update the FIBs of both old and new AR. When the handoff procedure is over, the content consumer will send the Interest to the old AR that will redirect the Interest to the new location of the producer. The total handoff latency can be generated as follows:

$$
\begin{aligned}
L_{i b a} & =L_{p n}+L_{\text {par }}+L_{\text {onar }}+L_{\text {onar }}+L_{\text {car }}+L_{\text {ars }}+L_{\text {onar }} \\
& =l_{p n}+a \times L w l_{u p}+2 b \times L w_{u p}+a \times L w l_{\text {int }}+c \times L w_{\text {int }}+b \times L w_{\text {int }}
\end{aligned}
$$

The handoff latency of propose scheme (PMSS) can be express as (7). When producer completes the handoff between old AR and new AR, it sends its new prefix information in MI packet to the new access router and the new access router forward it to the known location of the immobile anchor. The immobile anchor broadcast the MI to update the intermediate routers in the restricted domain. Then a consumer can reissues pending Interest toward the new location of the content producer. The expression is as follows:

$$
\begin{aligned}
L_{p m s s} & =L_{p n}+L_{\text {par }}+2 L_{\text {ars }}+L_{c a r}+L_{\text {sar }} \\
& =l_{\text {pn }}+a \times L w l_{\text {nint }}+2 c \times L w l_{\text {nint }}+a \times L w l_{\text {int }}+c \times L w_{\text {int }}
\end{aligned}
$$

\subsection{Handoff signaling and packets delivery cost analysis}

Handoff signaling cost is the number of messages sent over the network during handoff processes. The smaller signaling cost the better performance of the proposed scheme. The size of data or any additional packets can be represented as $S_{\text {name }}$. The MBMA signaling cost (8), the producer sent a packet to update the server about new name prefix, a consumer sent a packet to query the server to obtain a forwarding hint. Then the consumer can forward the Interest through optimal path to the producer and received data via the same route. The packets delivery cost is presented in (9).

$$
\begin{aligned}
C_{\text {mba }} & =C_{\text {par }}+C_{\text {sar }}+C_{\text {sar }}+C_{\text {car }}+C_{\text {car }}+C_{\text {ars }} \\
& =S_{\text {upack }} \times(a+d)+S_{\text {qryrep }}(d+a)+S_{\text {int }} \times(a+c) \\
C_{m b a} & =C_{\text {par }}+C_{\text {car }}+C_{\text {sar }}+C_{\text {par }}+C_{\text {car }}+C_{\text {sar }} \\
& =S_{\text {int }} \times(2 a+c)+S_{\text {data }} \times(2 a+c)
\end{aligned}
$$

The LBMA signaling cost (10) is formulated when producer sent a packet to update the new and old AR about new domain prefix, a consumer sent an Interest towards the old AR. Then old AR forwards the Interest with the location to the new AR in triangular passion, the data is also routed back to the consumer via the same route. The packets delivery cost is presented in (11). 


$$
\begin{aligned}
C_{\text {lba }} & =C_{\text {par }}+C_{\text {onar }}+C_{\text {car }}+C_{\text {sar }}+C_{\text {onar }}+C_{\text {car }} \\
& =S_{\text {upack }} \times(a+b)+S_{\text {int }} \times(a+c)+S_{\text {locint }} \times(b+a) \\
C_{\text {lba }} & =C_{\text {par }}+C_{\text {car }}+C_{\text {onar }}+C_{\text {sar }}+C_{\text {par }}+C_{\text {car }}+C_{\text {onar }}+C_{\text {sar }} \\
& =S_{\text {int }} \times(2 a+b+c)+S_{\text {data }} \times(2 a+b+c)
\end{aligned}
$$

The CDBMA signaling cost (12) is formed in a similar passion with MBMA, it also provides optimal [1] packet delivery path. The only difference is the server can update the entire FIB of the ARs within the domain. The packets delivery cost is presented in (13).

$$
\begin{aligned}
C_{c d b a} & =C_{\text {par }}+C_{\text {sar }}+C_{\text {sar }}+C_{\text {sar }}+C_{\text {car }}+C_{\text {ars }} \\
& =S_{\text {regack }} \times(a+d)+S_{\text {qfibup }} \times(2 d)+S_{\text {int }} \times(a+c) \\
C_{c d b a} & =C_{\text {par }}+C_{\text {car }}+C_{\text {sar }}+C_{\text {par }}+C_{\text {car }}+C_{\text {sar }} \\
& =S_{\text {int }} \times(2 a+c)+S_{\text {data }} \times(2 a+c)
\end{aligned}
$$

The IBMA signaling cost (14) is formulated in the same manner with LBMA, the only difference is an encapsulation of Interest packets between old and new AR. The packets delivery cost is presented in (15) which is also triangular routing path.

$$
\begin{aligned}
C_{i b a} & =C_{\text {par }}+C_{\text {onar }}+C_{\text {car }}+C_{\text {sar }}+C_{\text {onar }}+C_{\text {car }} \\
& =S_{\text {upack }} \times(a+b)+S_{\text {int }} \times(a+c)+S_{\text {encInt }} \times(b+a) \\
C_{\text {iba }} & =C_{\text {par }}+C_{\text {car }}+C_{\text {onar }}+C_{\text {sar }}+C_{\text {par }}+C_{\text {car }}+C_{\text {onar }}+C_{\text {sar }} \\
& =S_{\text {int }} \times(2 a+b+c)+S_{\text {data }} \times(2 a+b+c)
\end{aligned}
$$

The PMSS signaling cost (16) is formed when the producer sent a mobility packet to update the anchor, the anchor broadcast the mobility Interest to update the intermediate routers. A consumer can send an Interest and received data via the optimal route. The packets delivery cost is presented in (17).

$$
\begin{aligned}
C_{\text {pmss }} & =C_{\text {par }}+C_{\text {sar }}+C_{\text {sar }}+C_{\text {car }}+C_{\text {sar }} \\
& =S_{\text {mobInt }} \times(a+2 c)+S_{\text {int }} \times(a+c) \\
C_{\text {pmss }} & =C_{\text {par }}+C_{\text {car }}+C_{\text {sar }}+C_{\text {par }}+C_{\text {car }}+C_{\text {sar }} \\
& =S_{\text {int }} \times(2 a+c)+S_{\text {data }} \times(2 a+c)
\end{aligned}
$$

\section{IMPLEMENTATION}

\subsection{Model design and implementation}

The formulated models for handoff latency and signaling cost analysis is designed and implemented using Python programming in the Spyder IDE environment. The Spyder IDE is one of powerful development tool for python language that provides IPython console with an interactive prompt to execute codes. The analytical model was verified at the implementation phase to ensure the correctness and accuracy of programming codes ready for implementation design. The model was validated using parameters stated in Table 1, to observe and validate the accuracy of the model's applicability related to producer mobility problems such as handoff latency and cost, for all the driven analytical models of existing and proposed schemes.

\subsection{Algorithms}

The analytical model is implemented in two different algorithms. Algorithm one was implemented to execute the handoff latency, the signaling and data delivery cost for MBMA, LBMA, CDBMA, IBMA and proposed PMSS respectively. 
ALGORITHM ** Handoff Latency, Signalling Cost and Packets Delivery**

1. Begin

2. Declare variables: $B_{w}, B_{w l}, L d_{w}, L d_{w l}, Q_{d}$,

$a, b, c, d, M B M A, L B M A, C D B M A, I B M A, P M S S$;

3. Initialize variables: $B_{w}=100, \mathrm{~B}_{\mathrm{wl}=} 11, L d_{w}=2, L d_{w l} 10, Q_{d=} 5, a=1$;

4. Calculate

5. $\quad L w \_n a m e ~ \rightarrow\left(S \_n a m e /((B w+L d w+Q d))\right)$;

6. $\quad L w l \_n a m e \rightarrow((1+q) /(1-q)) \times\left(S_{-}\right.$name $\left./(B w l+L d w l)\right)$;

7. Begin

8. Let subscript name $\rightarrow$ up, int, nint

9. for $b$ in (5 to $\mathrm{n})$ do:

10. for $c$ in (5 to 23) do:

11. for $d$ in $(9$, to 27$)$ do:

12. Calculate Handoff_Latency (MBMA: LBMA: CDBMA: IBMA: PMSS)

13. Calculate Handoff_Signaling_Cost (MBMA: LBMA: CDBMA: IBMA: PMSS)

14. Calculate Data_Delivery_Cost (MBMA: LBMA: CDBMA: IBMA: PMSS)

15. End for;

16. End for;

17. End for;

18. Output ("b, c, d: HLatency of MBMA, LBMA, CDBMA, IBMA, PMSS");

19. Output ("b, $c$, d: SCost of MBMA, LBMA, CDBMA, IBMA, PMSS ");

20. Output ("b, $c$, d: DCost of MBMA, LBMA, CDBMA, IBMA, PMSS ");

21. End.

\section{RESULT ANALYSIS AND DISCUSSION}

For the numerical result analysis for both handoff latency and signaling cost, we set $a=1, b=c=5$ and $\mathrm{d}=9$ as used from the literature [16, 19], 16 bytes are used for additional field upon normal Interest packets as presented in Table 1. The numerical result on the handoff latency analysis have shown in Figure 3 (a), (b) and Figure 4 (a) that illustrate the effect of varying different transmission cost between old and new AR, ARs and server. In addition, compare the handoff latency of MBMA, IBMA, LBMA, CDBMA, and PMSS. In Figure 3 (a), the latency of IBMA and LBMA significantly increase by varying $b$, because a packet must route through old AR was also known as a home router. In Figure 3 (b), the latency of all approaches was increased significantly, due to the effect of varying cost between ARs that can directly affect any approach. In Figure 4 (a), the latency of MBMA and CDBMA significantly increase by varying $d$, and linearly for IBMA, LBMA, and PMSS as result of querying routing update from the server, control serves or resolution handler. Hence, we can realize that the handoff latency of propose scheme (PMSS) is the lowest with the effect of varying $b, c$, and $d$ in the midst of five different approaches.

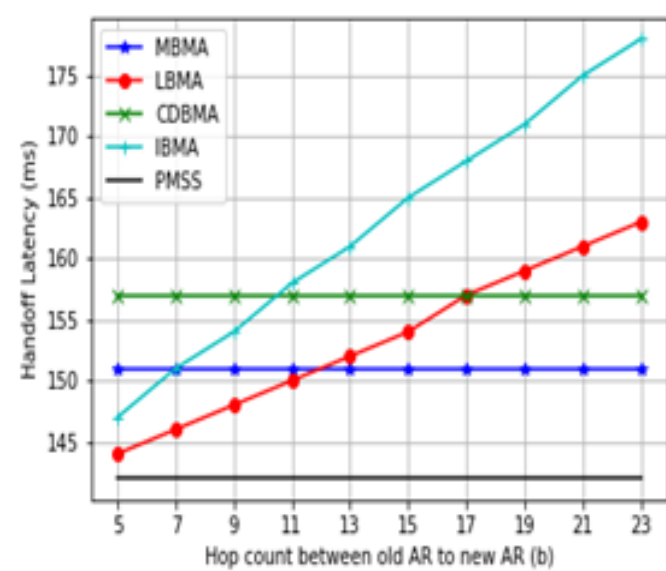

(a)

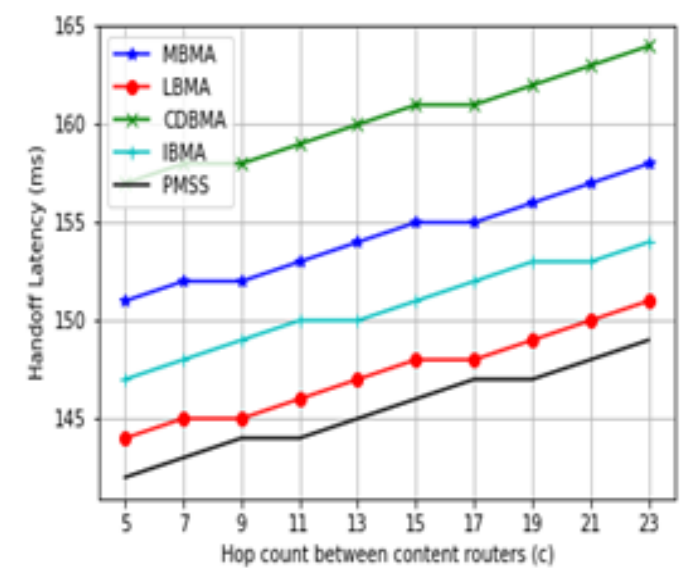

(b)

Figure 3. (a) Impact of b on handoff latency; (b) Impact of $c$ on handoff latency 


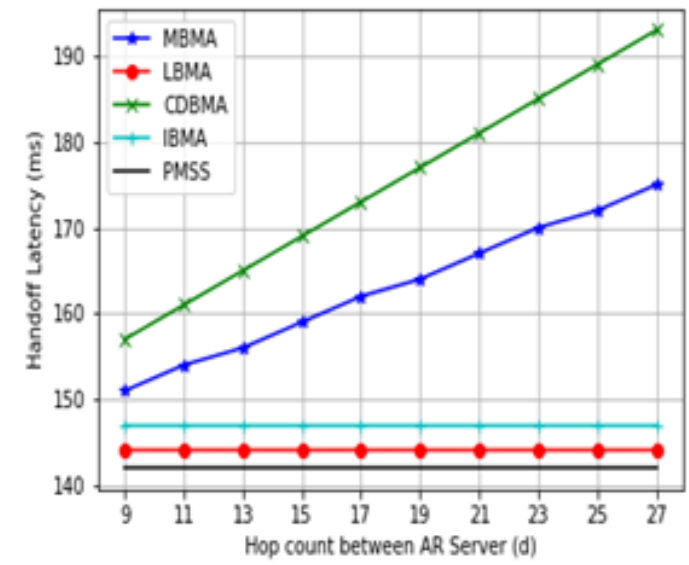

(a)

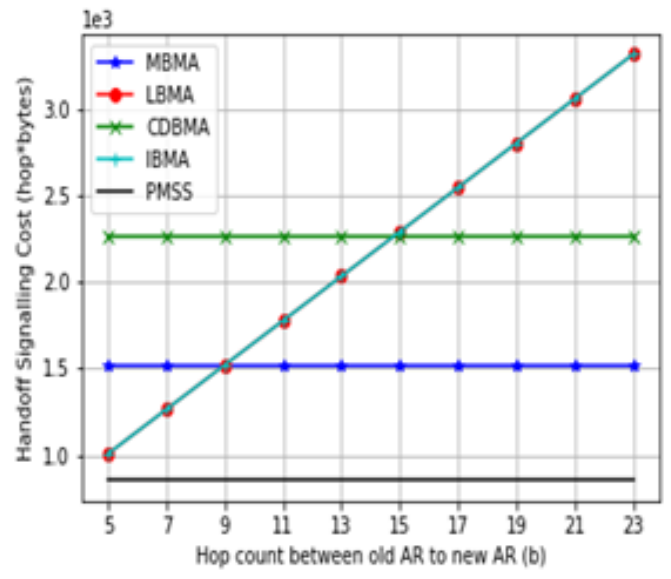

(b)

Figure 4. (a) Impact of d on handoff latency; (b) Impact of b on handoff signalling cost

The numerical result on the handoff signaling cost and optimal data delivery analysis have shown in Figure 4 (b), Figure 5 (a) and (b). The handoff latency and signaling cost are related based on the hop count analysis; there is certainty that when latency is high the signaling cost will also be high. From Figure 4 (b) we can see that the signaling cost of IBMA and LBMA are significantly increased, while for MBMA, CDBMA is uniform due to the increase of $b$ that directly affect the transmission between old and new AR. Moreover, Figure 5 (b) shows the signaling coat of all approaches increases significantly, due to the effect of varying $c$. In Figure 5 (b) the variation of $d$ affected only MBMA and CDBMA. Therefore, we can realize that the proposed scheme has the lowest signaling cost among five different approaches.

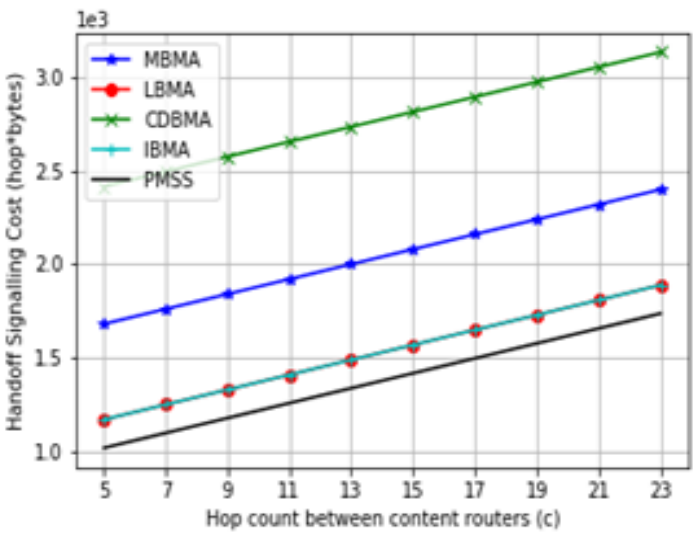

(a)

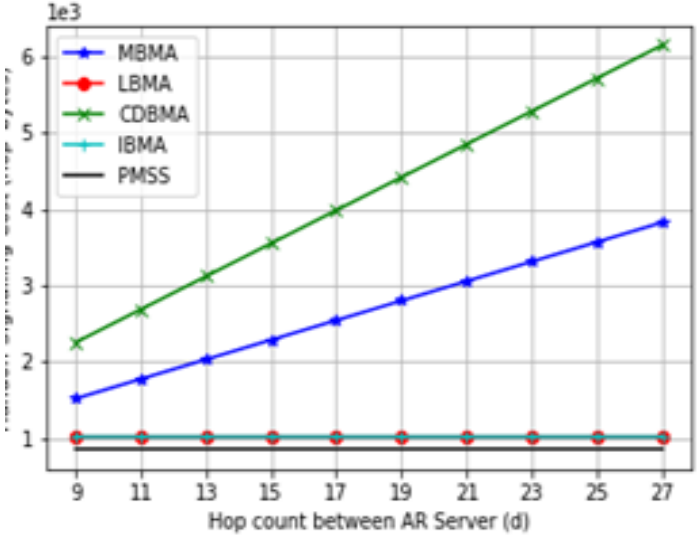

(b)

Figure 5. (a) Impact of c on handoff latency; (b) Impact of d on handoff signalling cost

Figure 6 (a) and (b) shows the optimal packets delivery for our proposed PMSS. In Figure 6 (a), the packets delivery of LBMA and IBMA are significantly increased, while that of MBMA, CDBMA, and PMSS are uniforms. Since from the literature, the approaches that use DNS-like server or control server transmitted their packages via optimal route after the handoff. Therefore, PMSS is optimal as it goes with MBMA and CDBMA. In Figure 6 (b), the variance of $\mathrm{c}$ affected all the approaches, hence PMSS and MBMA and CDBMA have the lowest cost. Therefore, we can conclude that PMSS is optimal in terms of packets delivery path. 


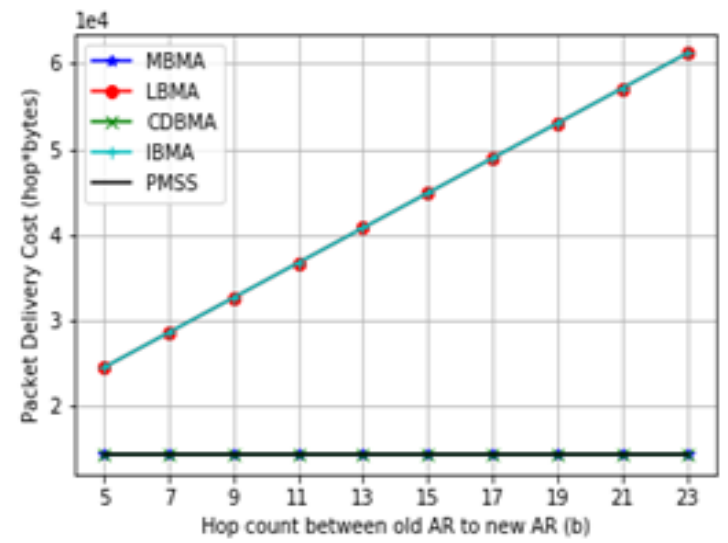

(a)

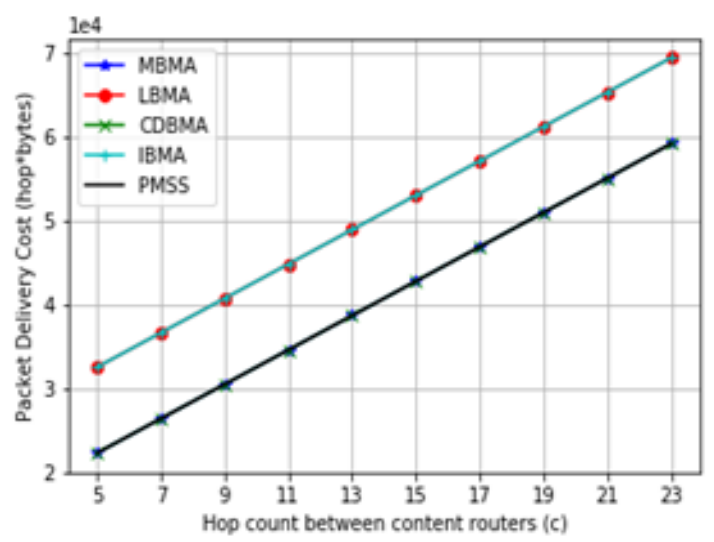

(b)

Figure 6. (a) Impact of $\mathrm{b}$ on packet delivery cost ;(b) Impact of $\mathrm{c}$ on packet delivery cost

\section{CONCLUSION AND FUTURE WORK}

The insufficient research on data path optimization after handoff causes serious degrading of seamless mobility support in NDN. In this paper, we have proposed a producer mobility support scheme that minimizes the handoff latency, signaling cost to reduce the unnecessary Interest packets loss. We introduce mobility Interest, broadcasting strategy and modified immobile anchor router to perform a dual function, that is anchoring and broadcasting. The numerical result shows that he PMSS reduces handoff latency, signaling overhead cost and improve data packets delivery via optimal path once a content producer relocates to another PoA compared to existing approach. Predominantly, handoff problems can be investigated using the analytical method, whereas other issues like the determination of packets delivery, packets losses, scalability and path optimization needs to be conducted through simulation investigation for a better result. Henceforth, in our future work, we will focus on simulation investigation would be carried out for the overall evaluation of handoff performance, path optimization, and packets delivery compared to specific existing schemes.

\section{REFERENCES}

[1] M. Hussaini, S. A. Nor, and A. Ahmad, "Producer mobility support for Information Centric Networking approaches : A review," Int. J. Appl. Eng. Res., vol. 13, no. 6, pp. 3272-3280, 2018.

[2] M. Hussaini, S. A. Nor, and A. Ahmad, "Producer mobility support scheme for Named Data Networking: A survey," International Journal of Electrical and Computer Engineering (IJECE), vol. 8, no. 6, pp. 31-42, 2018.

[3] I. Angri, M. Mahfoudi, A. Najid, and M. El Bekkali, "Exponential MLWDF (EXP-MLWDF) Downlink Scheduling Algorithm Evaluated in LTE for High Mobility and Dense Area Scenario," Int. J. Electr. Comput. Eng., vol. 8, no. 3, pp. 1618-1628, 2018.

[4] A. Ramli, N. Zulkifli, and S. M. Idrus, "Power consumption modeling and analysis of integrated optical-wireless access network," International Journal of Electrical and Computer Engineering (IJECE), vol. 7, no. 6, pp. 34753483, 2017.

[5] A. J. Jabir, "Bulk binding approach for PMIPv6 protocol to reduce handoff latency in IoT," International Journal of Electrical and Computer Engineering (IJECE), vol. 9, no. 3, pp. 1894-1901, 2019.

[6] A. Mohanty, S. Mahapatra, and U. Bhanja, "Traffic congestion detection in a city using clustering techniques in VANETs," vol. 13, no. 3, pp. 884-891, 2019.

[7] L. Zhang et al., "Named Data Networking," ACM SIGCOMM Comput. Commun. Rev., vol. 44, no. 3, pp. 66-73, 2014.

[8] L. V. Yovita and N. R. Syambas, "Caching on named data network: A survey and future research," International Journal of Electrical and Computer Engineering (IJECE), vol. 8, no. 6, pp. 4456-4466, 2018.

[9] M. A. Naeem, S. A. Nor, S. Hassan, and B. S. Kim, "Performances of probabilistic caching strategies in content centric networking," IEEE Access, vol. 6, no. c, pp. 58807-58825, 2018.

[10] B. Feng, H. Zhou, and Q. Xu, "Mobility support in Named Data Networking: a survey," Eurasip J. Wirel. Commun. Netw., vol. 2016, no. 1, 2016.

[11] D. Saxena, V. Raychoudhury, N. Suri, C. Becker, and J. Cao, "Named Data Networking: A Survey," Comput. Sci. Rev. Elsevier, vol. 19, pp. 15-55, 2016.

[12] F. Hermans, E. Ngai, and P. Gunningberg, "Global Source Mobility in the Content-Centric Networking Architecture," in Proceedings of the 1st ACM workshop on Emerging Name-Oriented Mobile Networking Design Architecture, Algorithms, and Applications - NoM '12, pp. 13-18, 2012. 
[13] Z. Zhu, A. Afanasyev, and L. Zhang, "A new perspective on mobility support," Named-Data Networking Project, Technical Report NDN-0013, pp. 1-6, 2013.

[14] M. Hussaini, S. A. Nor, A. Ahmad, I. S. Maijama'a, A. Isah, and A. Aminu, "Mobility support and operation of Information Centric Networking approach," Int. J. Comput. Netw. Inf. Secur., vol. 10, no. 10, pp. 18-25, 2018.

[15] Y. Rao, D. Gao, and H. Luo, "NLBA: A novel provider mobility support approach in mobile NDN environment," in IEEE 11th Consumer Communications and Networking Conference, CCNC 2014, 2014, no. CCNC, pp. 188-193, 2014.

[16] Y. Rao, H. Luo, D. Gao, H. Zhou, and H. Zhang, "LBMA: A novel Locator Based Mobility support Approach in Named Data Networking," China Commun., vol. 11, no. 4, pp. 111-120, 2014.

[17] G. Tyson, N. Sastry, R. Cuevas, I. Rimac, and A. Mauthe, "A survey of mobility in Information-Centric Networks," Commun. ACM, vol. 56, no. 12, pp. 90-98, 2013.

[18] M. Hussaini, S. A. Nor, and A. Ahmad, "PMSS : Producer mobility support scheme optimization with RWP mobility model in Named Data Networking," Int. J. Commun. Networks Inf. Secur., vol. 10, no. 2, pp. 329-339, 2018.

[19] T. X. Do and Y. Kim, "Optimal provider mobility in large-scale Named- Data Networking," KSII Trans. Internet Inf. Syst., vol. 9, no. 10, pp. 4054-4071, 2015.

[20] A. Chaabane, E. De Cristofaro, M. A. Kaafar, and E. Uzun, "Privacy in content-oriented networking:Threats and contermeasures," ACM SIGCOMM Comput. Commun. Rev., vol. 43, no. 3, pp. 25, 2013.

[21] G. White and G. Rutz, "Content Delivery with Content- Centric Networking," CableLabs, Strategy \& Innovation, no. February, pp. 1-26, 2016.

[22] M. Meddeb, A. Dhraief, A. Belghith, T. Monteil, and K. Drira, "Producer Mobility support in Named Data Internet of Things Network," Procedia Comput. Sci., vol. 109, pp. 1067-1073, 2017.

[23] S. Gao and H. Zhang, "Scalable mobility management for content sources in Named Data Networking," 201613 th IEEE Annu. Consum. Commun. Netw. Conf. CCNC 2016, pp. 79-84, 2016.

[24] A. Afanasyev et al., "NDNS: A DNS-like name service for NDN," 2017 26th Int. Conf. Comput. Commun. Networks, ICCCN 2017, no. Section II, 2017.

[25] X. Jiang, J. Bi, and Y. Wang, "What benefits does NDN have in supporting mobility," Proc. - Int. Symp. Comput. Commun., 2014.

[26] D. Kim, J. Kim, Y. Kim, H. Yoon, and I. Yeom, "End-to-end mobility support in Content Centric Networks," Int. J. Commun. Syst., vol. 28, no. 6, pp. 1151-1167, Apr. 2015.

[27] L. Rui, S. Yang, and H. Huang, "A producer mobility support scheme for real-time multimedia delivery in Named Data Networking," Multimed. Tools Appl., vol. 77, no. 4, pp. 4811-4826, 2018.

[28] Z. Yan, G. Geng, S. Zeadally, and Y. J. Park, "Distributed All-IP Mobility Management Architecture Supported by the NDN Overlay," IEEE Access, vol. 5, no. c, pp. 243-251, 2017.

[29] J. Lee, S. Cho, and D. Kim, "Device mobility management in Content-Centric Networking," IEEE Commun. Mag., vol. 50, no. 12 , pp. 28-34, 2012.

[30] F. Hermans, E. Ngai, and P. Gunningberg, "Mobile sources in an Information-Centric Network with hierarchical names: An indirection approach," in National Computer Networking, 2011.

[31] D. Han, M. Lee, K. Cho, T. Kwon, and Y. Choi, "Publisher mobility support in content centric networks," Int. Conf. Inf. Netw. 2014, no. February 2014, pp. 214-219, 2014.

[32] D. Han, M. Lee, K. Cho, T. T. Kwon, and Y. Choi, "PMC: Publisher Mobility Support for Mobile Broadcasting in Content Centric Networks," ASIA Futur. Internet 2012 Summer Sch. Kyoto, Japan, August. 2012., pp. 214-219, 2012.

[33] Z. Yan, S. Zeadally, S. Zhang, R. Guo, and Y.-J. Park, "Distributed mobility management in Named Data Networking," Wirel. Commun. Mob. Comput., vol. 16, no. 13, pp. 1773-1783, Sep. 2016.

[34] R. A. Rehman and B.-S. Kim, "LOMCF: Forwarding and Caching in Named Data Networking-based MANETs," IEEE Trans. Veh. Technol., vol. 66, no. 10, pp. 1-1, 2017.

[35] A. Azgin, R. Ravindran, and G. Wang, "On-demand mobility support with anchor chains in Information Centric Networks," IEEE Int. Conf. Commun., 2017.

[36] Z. Zhou, X. Tan, H. Li, Z. Zhao, and D. Ma, "MobiNDN: A mobility support architecture for NDN," in Proceedings of the 33rd Chinese Control Conference, CCC 2014, pp. 5515-5520, 2014.

[37] J. Tang, H. Zhou, Y. Liu, H. Zhang, and D. Gao, "A source mobility management scheme in Content-Centric Networking," 2014 IEEE 11th Consum. Commun. Netw. Conf. CCNC 2014, no. Ccnc, pp. 176-181, 2014.

[38] F. Ren, Y. Qin, H. Zhou, and Y. Xu, "Mobility management scheme based on Software Defined Controller for Content-Centric Networking," in Proceedings - IEEE INFOCOM, vol. 2016-Septe, no. 2, pp. 193-198, 2016.

[39] J. V. Torres, I. D. Alvarenga, R. Boutaba, and O. C. M. B. Duarte, "An autonomous and efficient controller-based routing scheme for networking Named-Data mobility," Comput. Commun., vol. 103, pp. 94-103, 2017.

[40] Y. Zhang, Z. Xia, S. Mastorakis, and L. Zhang, "KITE: Producer mobility support in Named Data Networking," in ICN '18: 5th ACM Conference on Information-Centric Networking (ICN '18), pp. 1-12, 2018.

[41] M. Hussaini, M. A. Naeem, B.-S. Kim, and I. S. Maijamara, "Efficient producer mobility management model in Information-Centric Networking," IEEE Access, pp. 1-21, 2019. 


\section{BIOGRAPHIES OF AUTHORS}

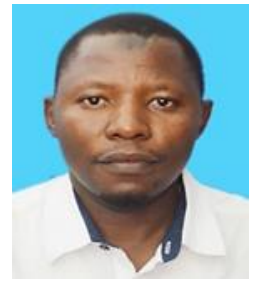

Muktar Hussaini: Received the B.S. degrees in Computer Science from the Usman Danfodiyo University Sokoto, Nigeria, in 2005, M.S. degree in Computer and Information Engineering, at International Islamic University Malaysia, in 2012. He is currently pursuing the Ph.D. degree in Computer Science at Universiti Utara Malaysia, Malaysia. He is currently a research student under the Internetwork Research Laboratory, School of Computing, Universiti Utara Malaysia, also a member of Internet Society and Nigerian Computer Society. From 2006, he worked with the Department of Computer Science at Hussaini Adamu Federal Polytechnic, Kazaure Jigawa State, Nigeria. His research interest includes the energy efficiency of wireless sensor networking, wireless sensor protocol and applications, future Internet research, Information Centric networking (ICN), Named Data networking (NDN), mobility support for ICN and producer mobility support for NDN,(e-mail: elhussenkaz@yahoo.com)

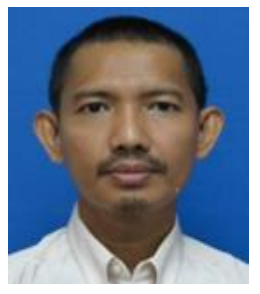

Shahrudin Awang Nor: Obtained his Master's degree in Computer and Information Networks (2000), at the University Of Essex United Kingdom and Ph.D. in Computer Network (2012) at Universiti Utara Malaysia. Now is currently a Senior Lecturer at the School of Computing, Universiti Utara Malaysia. Also, a researcher with the InterNetWorks Research Laboratory, College of Arts and Sciences, Universiti Utara Malaysia (UUM), is a member of Internet Society and IEEE member. (e-mail: shah@uum.edu.my ).

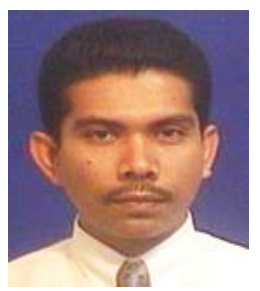

Amran Ahmad: Obtained his master's degree in Information Technology (2003), and Ph.D. Information Technology (2015) at Universiti Utara Malaysia. Now is currently a Senior Lecturer at School of Computing, Universiti Utara Malaysia. Also, a researcher with the InterNetWorks Research Laboratory, College of Arts and Sciences, Universiti Utara Malaysia (UUM), is a member of Internet Society and IEEE member (e-mail: shah@uum.edu.my ). 